\title{
Study on the Road Network Connectivity Reliability of Valley City Based on Complex Network
}

\author{
Yong-Sheng Qian, ${ }^{1}$ Min Wang, ${ }^{1}$ Hong-Xia Kang, ${ }^{2}$ \\ Jun-Wei Zeng, ${ }^{1}$ and Yu-Fei Liu' \\ ${ }^{1}$ School of Traffic and Transportation, Lanzhou Jiaotong University, Lanzhou 730070, China \\ ${ }^{2}$ Taiyuan Railway Bureau Party School, Taiyuan 030013, China \\ Correspondence should be addressed to Yong-Sheng Qian, qianyongsheng@mail.lzjtu.cn
}

Received 8 May 2012; Revised 30 August 2012; Accepted 10 September 2012

Academic Editor: Wuhong Wang

Copyright (C) 2012 Yong-Sheng Qian et al. This is an open access article distributed under the Creative Commons Attribution License, which permits unrestricted use, distribution, and reproduction in any medium, provided the original work is properly cited.

Based on the research progress in related fields and the distribution characteristics of road networks in valley cities, the complex network model of a city road network is established to study its connectivity reliability. Taking Lanzhou as the example, several parameters of the complex network abstracted from the road network are calculated and the practical meanings of them are described, respectively. On this basis, through computing the global efficiency and the relative size of the largest connecting subgraph under intentional attacks and random attacks, respectively, the curves of the above two parameters varying with the attacking times are drawn. The detailed investigation of connectivity reliability of Lanzhou road network is done by analyzing the curves' tendency. Finally, we find that the network of a valley city has a poor connection and has a lot of dead ends. Besides, the average length of the roads is very long and the holistic connectivity reliability is at a lower level; these are suitable to the group-type distribution of valley city's road network, and the connectivity reliability of the road network is stronger under random attacks than that under intentional attacks.

\section{Introduction}

Since the reform and opening up of China, the urbanization process has been accelerated greatly, which requires the urban road system to meet the requirements of the rapid development of cities. However, due to the limitations of historical and natural conditions, in many cities, especially the west valley cities, the phenomenon of urban road construction not meeting the requirements of urban development has appeared, which has brought inconveniences to the people's production and life travel and has led to a series of social 
problems. Lanzhou, as the transportation hub and logistic center in northwest China, is suffering from particularly serious transport problems. Study on the reliability of urban road network will be able to provide theoretical supports for pertinently changing city traffic congestion.

\section{Research Status in the Related Field}

Complexity science is the further development, enrichment, and deepening of systematic science and nonlinear science, and is the latest and the most forward area of scientific research. The complex network method, developed in recent years, has provided a new perspective for the research of the complexity of a system. Two articles [1,2] of complex network, published in Nature and Science in 1998 and 1999, respectively, pointed out that the connectivity distribution for many complex networks in real-world possesses exponential forms, and named such networks with scale-free property as scale-free networks. Since then, a boom of the study has been set off on complex network structure and its dynamical behavior, and the discussion on complex networks has penetrated into biology, physics, economics, computer science, as well as traffic transportation and other areas, which has improved the scientific understanding about the real world [3-9]. Jasny et al. used the complex theory to describe the connections in our life and science, he finds that the network analysis will help us to understand the world to a great degree [10]. Cho studied interactions between different people [11]. Bohannon studied the connections in some terrorist organizations, and helps the government to find out the leader of the terrorist organizations [12,13]. With that, the characteristics and dynamic features of complexity networks are researched by many scholars [14-18].

Connectivity was first proposed by Mine and Kawai in 1982, which reflects the probability to maintain connectivity between nodes in transport networks. Asakura puts forward the concept of travel time reliability in 1991, which is another measure method of network reliability, fully taking into account the travel needs of the road network and traveler behavior [19]. In 1994, Nicholson and Du defined the recession reliability of the traffic flow [20], namely the probability for the decline of OD pair or the traffic flow in the network not exceeding a certain value, and pointed out that one or more road network OD pairs will be directly affected when recession occurs in one road section. Lam and Zhang proposed the conception of demanding satisfaction reliability in 2000, which reflects the ability of providing the potential travel demand for road network, and describes the probability for the travel demand satisfaction rate not less than a particular value [21]. In 2002, Bell and Schmoker expanded the travel time reliability to the travel cost reliability [22], including travel time, travel distance, vehicle charges, and public transport costs; if the cost for completing a trip is less than a given threshold value, the corresponding road section is believed to be reliable. In China, based on the growth rules of the simulating tree, Zhu et al. proposed the channel generation method [23], of which all channels are efficient and suitable for large-scale network, discussed the effective channels, sensitivity of road sections and the reliability; for the fallibility factors of the road traffic network, Jin explored the reliability of the evaluation system of urban road traffic networks, and researched the redundancy and the economy of time and space of road transport network from the viewpoint of dynamic degeneration [24]. In 2005, Lin et al. studied the reliability of transport networks in emergencies and gave out the three probability problems usually considered in unexpected situations: the destruction of properties of nodes and sections, repair characteristics of nodes and sections, and the connectivity reliability in emergencies 
[25]. Fan et al. gave out the evaluation indexes of network reliability and the calculating model of reliability based on the network topology [26], and determined the arc, path, node, and the calculation method of reliability of the network structure; Yuan et al. considered the state of uncertainty of the transportation network under realistic traffic conditions [27], and proposed the change of choice of behavior from uncertain to deterministic network state and the route choice of model based on travel time reliability.

Through the analysis of the literature, we can see that the study of reliability of road network has transformed from the study only considering the physical structure of the road network to that of consideration of loading traffic flow and the mutual influence between travel demand and the capacity of road network, as well as the travel behavior of road users; the corresponding reliability indexes are connectivity reliability and travel time reliability. During this period, some scholars have proposed demand satisfaction reliability, the weak point reliability, performance index reliability, and suffered reliability. In China, research is conducted on the basis of foreign studies, and is the supplement or continuation of foreign study results; there is no significant breakthrough in the road network reliability indexes, and most of the researches are on connectivity reliability and travel time reliability. But domestic researches have explored the connectivity reliability and travel time reliability from different perspectives and conditions, which widen the thoughts and provide a foundation for further study on this area. There are also scholars that have studied the road network capacity reliability.

It can be seen from the results in the field that research on transport network reliability is increasing every year, which reflects the increasing travel demand in daily lives and higher requirements proposed by travelers on the travel system. In this paper, we studied the connectivity reliability of the valley city road networks using the theory of complex network.

\section{Definition of Valley City and Its Urban Road Network Distribution Characteristics}

From the perspective of geography, considering the location of the city and the relationship between the city and the valley, we define the valley city as follows: a city with the urban built-up area or the main or core part of the built-up area located in a valley, and the development of the main body of the city is strongly directly restricted by the valley terrain and the surrounding mountains or hills [27]. Because of the strong direct restriction by the valley terrain and the surrounding mountains or hills, the urban road network generally shows the banded distribution characteristic.

Compared with a plain city, the valley city road network presents its unique features. Firstly, with the limitation of the mountains and rivers, and in order to reduce the slope of the road, the valley city road lines are often built along the mountains or rivers, and road network form is always the freestyle. The valley city road lines are adapted to the natural terrain in order to save road engineering cost. Most of the road is not a straight line, and the road network is irregular. The valley city road network makes it difficult to form the ring road. Another big characteristic of a valley city is that the transportation is mainly concentrated on the main road of the urban axis. With the influence of the banded terrain and the problems of shortage of land, there is not enough land space to expand the urban road and share the traffic pressure when the urban trunk road is saturated. To research the connectivity reliability of valley city road network we will provide the reference to urban road network design and construction. 


\section{The Construction of the Complexity Network of Valley City Road Network}

The complexity network can be described accurately using the language and symbols of the graph theory. The findings and techniques in graph theory about network have been widely transplanted into complexity networks. Based on this, the urban road network model has been established based on complexity network.

For the urban road network, intersections of the city's road network can be abstracted as nodes, and road sections connecting the intersections can be abstracted as edges in the network. Therefore, the city's road network can be abstracted as the complex network composed by intersection and road sections connecting the intersections.

The following assumptions have been made when constructing the complex network to simplify the issue.

(1) If from intersection A in the urban road network, we can get to intersection B through a certain road section, and through the same road section, we can get to intersection A from intersection B, we consider the above connections as the same one, namely, implementing undirected treatment on the network.

(2) Ignore the actual length of the road sections that connect nodes of the urban road network. Assume that length of road sections are all 1, then the distance between nodes can be expressed as the number of road sections between the nodes, which do not consider the weights of the connections, and abstract the urban road network as a nonweighted network.

(3) In this work, residential districts at road sections, river crossing transport facilities, and ends of roads are considered as network nodes, which are equivalent to intersections. At the same time, if there is more than one road section connecting two nodes, then consider these sections as one connection, which is only one side between the two nodes. Consider road sections connecting two intersections directly in a reality network as sides with length = 1 in the model; from any intersection in the network, we can get to any other intersection, namely, the entire network is connected.

\section{Study on the Road Network Connectivity Reliability of Lanzhou}

\subsection{Definition of Urban Road Network Connectivity Reliability under Complex Network Theory}

Based on the definition of the system reliability and the temporal and spatial imbalance of the urban transport travel, this paper presents the general definition of the urban road network reliability as: the statistical probability for the urban road network meeting a particular traffic travel demand under different spatial and temporal distribution.

Urban road network connectivity reliability based on the complex network is defined as follows: the ability for urban road network to maintain the connectivity state, after being attacked in different ways and the network suffering from a certain level of destruction.

\subsection{Instructions of Parameters in Valley City Road Network Model}

\subsubsection{Degree and Degree Distribution}

The degree $k_{i}$ of intersection (node) $i$ is the total number of the road sections connecting intersection $i$; the average value of the degrees of all intersections is defined as the average 
value of the urban road network degree; the degree distribution is indicated with function $p(k)$, reflecting the probability for a random intersection being connected with $k$ road sections. The degree of the urban road network is the number of intersections connecting an intersection directly. The degree distribution reflects the maturity degree of the urban road network; the high degree distribution indicates that the urban road network has high network rate and less dead ends, and relatively high reliability.

\subsubsection{Average Path Length}

In complex networks, the distance $d_{i j}$ between nodes $i$ and $j$ is defined as the minimum number of the sides connecting the two nodes; the network diameter is defined as the largest distance of two random nodes; the average value of the distances of all the node pairs of the complex networks is marked with $L$, which reflects the degree of separation between nodes and can be calculated by the following formula:

$$
L=\frac{1}{1 / 2 N(N+1)} \sum_{i \geq j} d_{i j}
$$

where $N$ is the node number. If we ignore the distance between node $i$ and itself because it is zero, the above equation becomes:

$$
L=\frac{1}{1 / 2 N(N-1)} \sum_{i>j} d_{i j}
$$

The path length of urban road network is the distance from one intersection to another and the average path length $L$ refers to the average value of the shortest path length between all intersection pairs. The small $L$ indicates a small distance between any two intersections in the road network, namely, good overall road network reachability.

\subsubsection{Clustering Coefficient}

The clustering coefficient is the characteristic parameter used to describe the tightness of the network, marked with $C$. A node with large clustering coefficient indicates that the tightness level of the adjacent nodes is high, and it is easy to form regional agglomeration for the subnetwork composed by this node and its neighboring nodes. It is calculated as follows: assume node $i$ in the network is connected with $k_{i}$ nodes through $k_{i}$ sides (if the $k_{i}$ nodes are connected to each other, then there should be $1 / 2 k_{i}\left(k_{i}-1\right)$ sides) and suppose that there are in fact only $E_{i}$ sides connected to each other within $k_{i}$ nodes, then the ratio between $E_{i}$ and $1 / 2 k_{i}\left(k_{i}-1\right)$ is the Clustering coefficient, which is:

$$
C_{i}=\frac{2 E_{i}}{k_{i}\left(k_{i}-1\right)} .
$$


The entire network's clustering coefficient $C$ is the average clustering coefficient of all nodes throughout the network, which is:

$$
C=\frac{1}{N} \sum_{i} C_{i}
$$

The clustering coefficient of the urban road network reflects the aggregation of an intersection and its $k_{i}$ adjacent intersections. The clustering coefficient is indicated with the ratio between the number of sections connecting the $k_{i}$ intersections and the probable total number of those sections. Clustering coefficient reflects the aggregation between an intersection and its $k_{i}$ adjacent intersections, and the great clustering coefficient represents the high level of short-range contact between intersections of the road network, namely, great density.

\subsubsection{Efficiency of the Network}

The efficiency of the network is qualified to analyze the network of small-world behavior instead of the average path length and clustering coefficient. The efficiency $\varepsilon_{i j}$ between nodes $i$ an $j$ of the network is the reciprocal value of the distance $d_{i j}$ between the two points, namely, $\varepsilon_{i j}=1 / d_{i j}$. If it is unreachable between nodes $i$ and $j$, then $d_{i j}$ tends to zero, and the corresponding $\varepsilon_{i j}=0$. For the entire network, the average value of efficiencies between all node pairs is defined as the global efficiency, denoted with $E_{\text {glob: }}$ :

$$
E_{\text {glob }}(G)=\frac{1}{N(N-1)} \sum_{i \neq j \in G} \varepsilon_{i j}=\frac{1}{N(N-1)} \sum_{i \neq j \in G} \frac{1}{d_{i j}} .
$$

Taking into account the situation of nonconnected graph, the local features of the network can be represented by the average efficiency of the partial subgraph $G_{i}$ (all nodes connecting with node $i) . E\left(G_{i}\right)$ can be used to analyze the effectiveness of the information transmission between node $i^{\prime}$ s adjacent nodes. $E_{\text {loc }}$ is corresponding to the clustering coefficient $C$. Consider the following:

$$
\begin{gathered}
E\left(G_{i}\right)=\frac{1}{k_{i}\left(k_{i}-1\right)} \sum_{i \neq m \in G_{i}} \frac{1}{d_{l m}}, \\
E_{\mathrm{loc}}(G)=\frac{1}{N} \sum_{i \in G} E\left(G_{i}\right) .
\end{gathered}
$$

For the urban road network, $G_{i}$ is the subgraph formed by the $k_{i}$ intersections adjacent to intersection $i$, the maximum number of road sections is $1 / 2 k_{i}\left(k_{i}-1\right)$, and $d_{l m}$ is the length of the shortest path between nodes $l$ and $m$ of subgraph $G_{i}$. The efficiency of the urban road network reflects the connectivity, that is, the density of the intersections. 


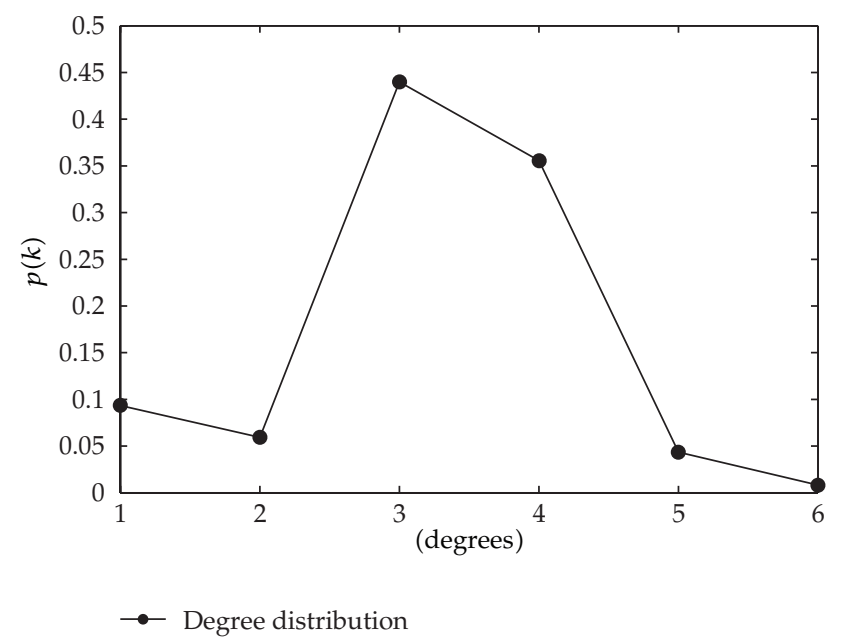

Figure 1: Degree distribution of Lanzhou City road network.

\subsubsection{The Relative Size of the Largest Connected Subgraph}

The largest connected subgraph of the network means the subgraph connecting all nodes with least sides. The relative size $S$ of the largest connected subgraph is an important amount, weighing the capacity maintaining the original function of a network when being attacked continuously, which is the ratio of the total node number of the largest connected subgraph $N^{\prime}$ and total node number of the network $N$ :

$$
S=\frac{N^{\prime}}{N}
$$

The relative size of the largest connected subgraph weighs the capacity maintaining the original function of a network after destruction.

\subsection{Static Statistical Eigenvalues of the Road Network in Lanzhou City}

In the study, the existing nodes and road sections of Lanzhou city were selected (a total of 349 selected intersections and 507 road sections). Calculating the degree and degree distribution of Lanzhou city road network, the average path length and the clustering coefficient is helpful to studying the topological properties of the Lanzhou city road network. Analyzing these statistical eigenvalues has a practical significance for learning the valley city road network and its connectivity reliability.

\subsubsection{Degree and Degree Distribution}

For the Lanzhou city road network model, node degree reflects the importance of the node in the network; its practical significance is the number of road sections connected to the intersection in different directions. Figure 1 shows the calculation results of degree distribution of Lanzhou City road network. 
It can be seen from Figure 1 that the largest degree value is 6, followed by the value of 5 , and these nodes share relatively smaller proportions. The reason why there are intersections connecting 5-6 road sections in different directions is that in the process of the network model handling, two close intersections are considered as one, including a number of important road junctions and overpasses. Combined with the actual situation of Lanzhou City, several overpasses and the Yellow River Bridges are connected closely, so the above treatment is done when handling nodes corresponding to these places. These nodes, corresponding to the Xiaoxihu overpass and the Jiefangmen overpass, have the greatest degree values, indicating that they are the most complex intersections in the road transport network in Lanzhou City.

Nodes with a degree $=3$ in the Lanzhou city road network account for more than $40 \%$ of the total nodes, indicating that junctions of three roads account for a large proportion in the Lanzhou city road network, this is because of the limitations of topographical, the trunk line distribution of Lanzhou City is usually along the rivers and mountains, and the secondary roads, slip roads connected to the trunk roads, which is very easy to form junctions of three roads. Nodes with a degree $=4$ account for the second largest proportion. No matter what type of urban road network, common road intersections are the convergence of four directions, which meets the actual situation; the grid distribution and the irrational square format local road network in Lanzhou result in a lot of crossroads, whose corresponding node degree value is 4 . Nodes with degrees $=1,2$ account for about $20 \%$ of the total nodes, and the number of nodes with a degree $=1$ is more than that with a degree $=2$, which equates with the situations of Lanzhou. Because the road network is not perfect in Lanzhou, there are a lot of cul-de-sac with a corresponding degree $=1$; in addition, city roads in Lanzhou show long strip distributions, and there are a lot of living areas distributed along the strip roads; the intersections of the living areas and the roads are considered as nodes with a degree $=2$. The above analysis shows that the model results are consistent with the actual situation.

\subsubsection{Average Path Length}

The path length of the network represents the minimum number of road sections from a node to the specified node. Corresponding to the urban road network in Lanzhou, its practical significance is the number of road sections from one intersection to the specified intersection. Figure 2 is the statistical chart of the average path length of the Lanzhou road network. The figure shows that the proportion of intersections with the average node path length less than or equal to 8 is less than $36 \%$, indicating that the average path length of the network is relatively large, and for most of the intersections, more than 8 road sections need to be passed to get to the other intersection in the network. The largest average path length of Lanzhou city road network is 27 , indicating that moving from any intersection to another, the maximum average number of road sections to pass is 27 . However, since the assumption of the length of all the road sections in the network model is 1 , the calculation results are different from the actual length while the number of sections to go through matches the actual. In this article, the statistics data we applied is the average path length of the road network, but for the actual situation, the path length between the intersections at the edge of the network will exceed 27 because the intersections at the edge are isolated from other intersections. In addition, the average path length of the urban road network in Lanzhou is 21.9, that is, there are 21-22 sections from one intersection to another in Lanzhou city road network. The large average 


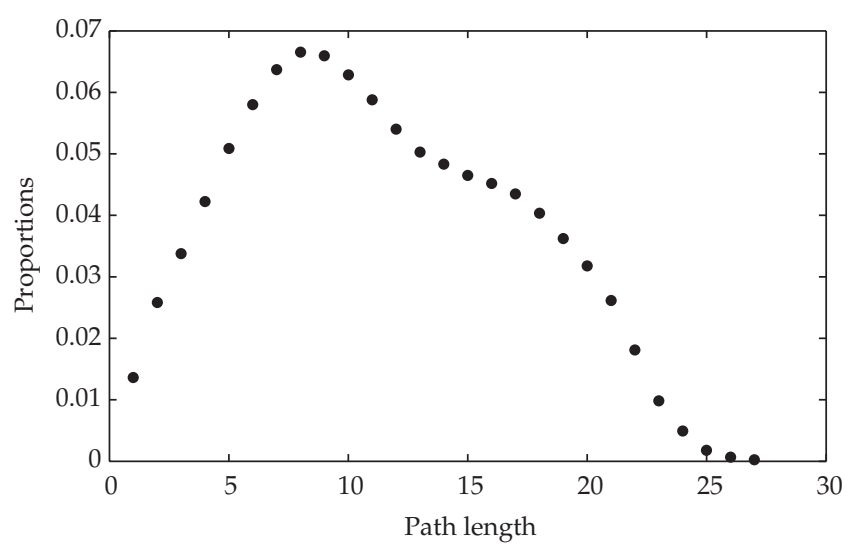

Figure 2: Statistical chart of the average path length of Lanzhou.

path length of the network has a direct relationship with the long strip and cluster layout of the Lanzhou road network, and the calculated results are consisted with the actual situation.

\subsubsection{Cluster Coefficient}

Another important statistical characteristic parameter of complex network is the clustering coefficient, which is a physical quantity measuring the density of the network between adjacent nodes. The practical significance of the clustering coefficient corresponding to the road network of Lanzhou is the aggregation level of intersections in Lanzhou. Figure 3 is the statistical chart of the clustering coefficient of the road network in Lanzhou. It can be seen from Figure 3 that intersections with clustering coefficient $=0$ account for $75.5 \%$ of the total, indicating that $75.5 \%$ of the intersections of Lanzhou are in a star-shaped framework, which the connections between the adjacent intersections must go across it. The number of nodes with clustering coefficient $=0$ is zero, indicating that no road section between adjacent nodes can achieve straight-through in Lanzhou road network. The overall clustering coefficient of the whole road network of Lanzhou is only 0.0582 , indicating a poor aggregation, which is consistent with the cluster and long strip distribution of the Lanzhou road network.

\subsection{Analysis of the Connectivity Reliability of Lanzhou Road Network}

Complex networks usually face two kinds of attacks: random attacks and deliberate attacks. As a small world network, the urban road network in Lanzhou is also facing these two attacks. This paper studies the changes of the connectivity reliability of Lanzhou road network under the two attacks. According to the actual situation of the urban road network in Lanzhou, the following specific assumptions were made for the two attack patterns.

\subsubsection{Assumptions for Random Attacks}

The random attack refers to nodes in the network being deleted randomly at a certain probability. Taking into account the relatively large number of intersections, each attack will 


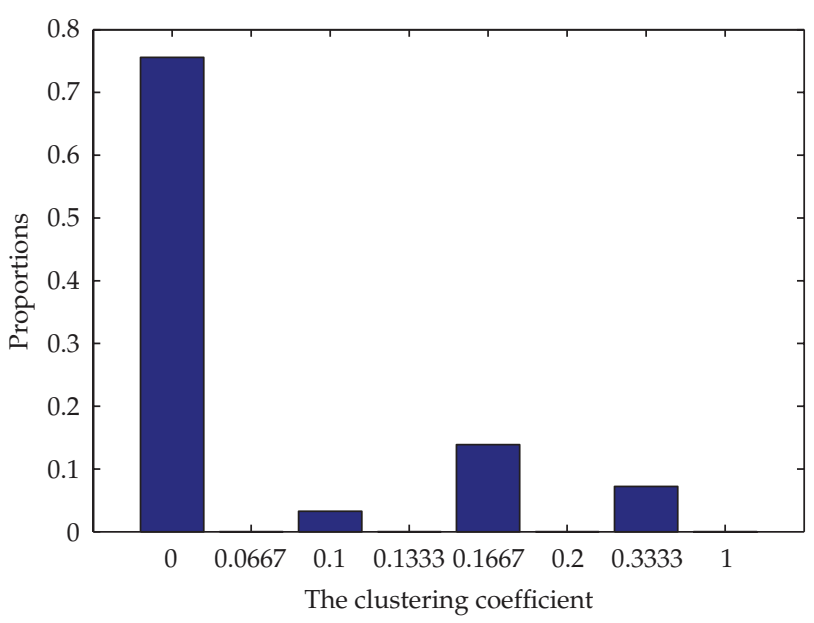

Figure 3: Statistical chart of the clustering coefficient of Lanzhou.

remove 5 nodes in the network, corresponding to 5 intersections of the urban road network in Lanzhou City.

\subsubsection{Assumptions for Deliberate Attacks}

The deliberate attack is the other attack mode the complex network suffered, which is a strategic attack on the nodes in the network. The purpose of this attack is to make the most severe damage with the least number of attacks. Therefore, this attack aims at and destroys the node with the greatest degree value of the network. In this work, the purpose of the deliberate attack is to make the most severe damage and make the road network of Lanzhou collapse as soon as possible. To make contrast with the random attack, 5 nodes with the greatest degree value will be removed from the network, corresponding to the 5 intersections with the most convergence.

\subsubsection{Analysis of Results}

Based on the above assumptions, the global efficiency $E_{\text {glob }}$ and the relative size of the network's largest connected subgraph $S$ under the two attacks are calculated in this paper. In order to more clearly describe the changes of the connectivity reliability of Lanzhou road network under different attack modes, the random attacks and deliberate attacks were compared, and the change curves of different indexes of the connectivity reliability based on the above data were formed. Specific analysis is as follows.

(1) Change curves of global efficiency $E_{\text {glob }}$ of Lanzhou road network.

The global efficiency is an important indicator measuring the overall road network connectivity of Lanzhou. The calculated Lanzhou road network global efficiency is 0.118 , indicating that the connectivity reliability of Lanzhou is poor.

Figure 4 shows the change curves of global efficiency under different attacks of Lanzhou road network. It can be seen from the figure that the global efficiency decreases faster under deliberate attacks compared with random attacks. 


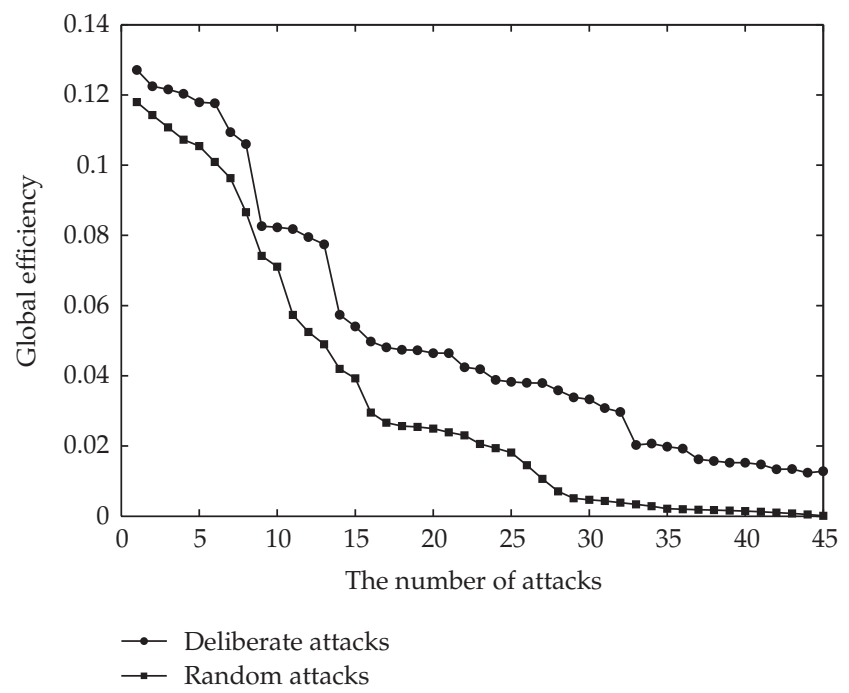

Figure 4: Change curves of global efficiency under different attacks.

Under deliberate attacks, the global efficiency will decrease to below 0.06 , which is only half of the original value, after 11 attacks (the deleted nodes only accounting for $15.7 \%$ of the total nodes); the network global efficiency will decrease to 0.02 after 23 attacks (deleted nodes accounting for $33 \%$ of the total nodes), indicating that the connectivity of the network reliability has become very poor; after 45 attacks (the deleted nodes accounting for $64.5 \%$ of the total nodes), the global efficiency of the network will become 0.00013 (close to 0 ), which shows the road network has been near-collapse. Under random attacks, after 14 attacks (the deleted nodes only accounting for $20 \%$ of the total nodes), the global efficiency will decrease to below 0.06; after 35 attacks (the deleted nodes accounting for $50.1 \%$ of the total nodes), the global efficiency will decrease to 0.02 , which means that the connectivity reliability will become poor when more than half of the nodes are attacked; after 45 attacks (the deleted node accounting for $64.5 \%$ of the total nodes), the global efficiency becomes 0.01279 , which is much larger than that of the deliberate attack (0.00013), and until this time, the Lanzhou road network has not been crashed.

The reason for these results is that there are great differences in the deletion of nodes under the two attacks. The deliberate attack is carried out in accordance with the degree value of the nodes. After the nodes with relatively larger degree value of the network, which play a very important role in maintaining the road network connectivity reliability, are removed, dramatic changes will occur on the topology fabric of the road network, leading to a great number of isolated intersections, and ultimately the collapse of the entire network. However, for random attacks, the probability for removing nodes according to the order of deliberate attacks is very small, and the network will demonstrate stronger robustness than that of deliberate attacks when subjected to random attacks, so more isolated intersections are needed to make the same extent of damage as the random attack. The above analysis shows that the damage on the connectivity reliability of the Lanzhou road network subjected to random attacks is slighter than that of deliberate attacks.

(2) Change curves of the relative size $S$ of the largest connected subgraph of Lanzhou road network. 


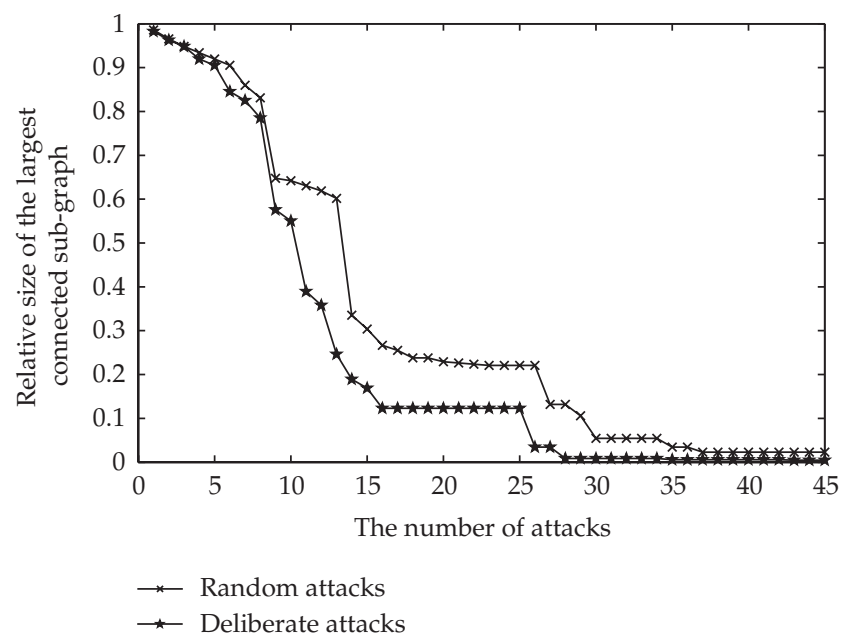

Figure 5: Change curves of relative size of the largest connected subgraph under different attacks.

Figure 5 shows the change curves of the relative size of the largest connected subgraph of Lanzhou road network subjected to different attacks under different times. It can be seen from the figure that the relative size of the largest network connected subgraph of the Lanzhou road network decreases faster under deliberate attacks compared with random attacks.

Under deliberate attacks, the relative size $S$ of the largest connected subgraph of the network decreases from 1 to 0.8252 with a small reducing extent and a slow speed after 7 attacks; $S$ decreases sharply from 0.8252 to 0.1232 during the 7 th to the 16 th attack; in the subsequent attacks, the speed of $S$ decrease is gradually slowing down, until the 45 th attack when $S$ is close to 0 . The above process shows that during the first 7 attacks, little intersections are attacked and isolated, and most of the nodes are maintained in the largest connected subgraph, namely the connectivity reliability can be maintained at a relatively high level; in the next $7-16$ attacks, the number of isolated intersections is gradually increasing, the number of nodes of the largest connected subgraph of the network is rapidly decreasing with the increasing number of attacks, the entire network has been divided into a number of relatively smaller networks with less nodes (maximum number of nodes in small networks is $12.32 \%$ of the original network), and the connectivity reliability has been very poor. In subsequent attacks, the subgraphs of Lanzhou road network are further divided, and the number of isolated intersections keeps increasing, until the entire network collapses. As for random attacks, the changes of the relative size of the largest connected subgraph show a similar trend as deliberate attacks; the difference is that the relative size of the largest connected subgraph under random attacks always maintains a higher level than that of deliberate attacks, and after the 45th attacks, it still remains at 0.0229, much larger than deliberate attacks of 0.0046 . This process shows that, for random attacks, the connectivity reliability will degenerate as deliberate attacks; however, the network will maintain a certain level of connectivity reliability (the largest connected subgraph of the network maintains nearly $86 \%$ of the intersections of the original network) under less attacks (7 times); with the increase of the attack number, the intersections of the largest connected subgraph will be reduced greatly, and then will gradually level off. 
The reason for these results is that when the network is subjected to deliberate attacks, the node removing is carried out in accordance with the degree value of the nodes; after the nodes with relatively larger degree value are removed during the early attacks, the network will be instantly differentiated into several subnets with sharply reduced nodes, so the relative size of the largest connected subgraph will change dramatically, the result of which is the maintenance of the degree of nodes left in the subgraphs at a very low level. When the network is subjected to random attacks, the deleting order is completely random, and the node with the largest degree value may remain in the largest connected subgraph until the end of the 45th attack; so in this case, the relative size of the largest connected subgraph may still have a relatively larger value compared with deliberate attacks. The above analysis shows that the connectivity reliability of the Lanzhou road network subjected to random attacks is better than that of deliberate attacks.

\section{Conclusions}

The paper constructs a complex network model of a valley city road network. Taking Lanzhou as an example of a typical valley city, the connectivity reliability of the road network was researched and the following conclusions are obtained.

(1) Valley city road network has poor connection and a lot of dead ends, and the number of road sections between intersections is too large, which makes it a poorly compacted network.

(2) Valley urban road network has long average path length and poor overall connectivity reliability, consistent with its cluster long strip layout; and the connectivity reliability of the urban road network subjected to random attacks is better than that of deliberate attacks, which is consistent with the characteristics of complex networks under attack.

\section{Acknowledgments}

This work is partly supported by the Humanities Social Sciences Programming Project of the Ministry of Education of China (no. 10YJA630126) and the State Social Science Fund Project (no. 11CJY067).

\section{References}

[1] D. J. Watts and S. H. Strogatz, "Collective dynamics of 'small-world' networks," Nature, vol. 393, no. 6684, pp. 440-442, 1998.

[2] A. L. Barabási and R. Albert, "Emergence of scaling in random networks," Science, vol. 286, no. 5439, pp. 509-512, 1999.

[3] D. Cui, Z. Y. Gao, and X. M. Zhao, "Cascades in small-world modular networks with 'CML's method," Physica B, vol. 17, pp. 1703-1710, 2007.

[4] Z. Zhi, F. Zhong-Qian, and Y. Gang, "Synchronization speed of identical oscillators on community networks," Chinese Physics B, vol. 18, no. 6, pp. 2209-2212, 2009.

[5] C. Di, G. Zi-You, and Z. Jian-Feng, "Properties of asymmetrically evolved community networks," Chinese Physics B, vol. 18, no. 2, pp. 516-521, 2009.

[6] H. F. Du, S. Z. Li, W. F. Marcus, Z. S. Yue et al., "The topologieal raileay network of China constrained by the geographic factors," Acta Physica Sinica, vol. 57, pp. 6771-6776, 2008 (Chinese).

[7] J. Li, B. H. Wang, P. Q. Jiang, T. Zhou, and W. X. Wang, "Growing complex network model with acceleratively increasing number of nodes," Acta Physica Sinica, vol. 55, no. 8, pp. 4051-4057, 2006 (Chinese) 
[8] M. Zhao, B. H. Wang, P. Q. Jiang et al., "Recent advancement in research of synchronization of dynamical systems on complex networks," Progress in Physics, vol. 25, pp. 273-295, 2005 (Chinese).

[9] Q. Luo, W. Wu, L. X. Li, Y. X. Yang, and H. P. Peng, "Adaptive synchronization research on the uncertain complex networks with time-delay," Acta Physica Sinica, vol. 57, no. 3, pp. 1529-1534, 2008 (Chinese).

[10] B. R. Jasny, L. M. Zahn, and E. Marshall, “Connections,” Science, vol. 325, no. 5939, p. 405, 2009.

[11] A. Cho, "Ourselves and our interactions: the ultimate physics problem?" Science, vol. 325, no. 5939, pp. 406-408, 2009.

[12] J. Bohannon, “Counterterrorism's new tool: 'Wletahetwork' analysis," Science, vol. 325, no. 5939, pp. 409-411, 2009.

[13] J. Bohannon, “Investigating networks: the dark side," Science, pp. 410-411, 2009.

[14] M. Kaiser and C. C. Hilgetag, "Optimal hierarchical modular topologies for producing limited sustained activation of neural networks," Frontiers in Neuroinformatics, vol. 4, p. 112, 2010.

[15] Y.-Y. Ahn, J. P. Bagrow, and S. Lehmann, "Link communities reveal multiscale complexity in networks," Nature, vol. 466, no. 7307, pp. 761-764, 2010.

[16] M. Newman, "The physics of networks," Physics Today, vol. 61, no. 11, pp. 33-38, 2008.

[17] A. L. Barabási, "Scale-free networks: a decade and beyond," Science, vol. 325, no. 5939, pp. 412-413, 2009.

[18] D. Garlaschelli, A. Capocci, and G. Caldarelli, "Self-organized network evolution coupled to extremal dynamics," Nature Physics, vol. 3, no. 11, pp. 813-817, 2007.

[19] Y. Asakura, "Reliability measures of an origin and destination pair in a deteriorated road net- work with variable flows," in Proceedings of the 4th Meeting of the EURO Working Group in Transportation, pp. 398-412, University of Newcastle, UK, 1996.

[20] A. J. Nicholson and Z. P. Du, "Improving transportation system reliability: a framework," in Proceedings of the 17th Australian Road Research Board Conference (ARRB '94), pp. 1-17, August 1994.

[21] W. H. K. Lam and N. Zhang, "A new concept of travel demand satisfaction reliability for assessing road network performance," in Proceedings of the Matsuyama Worshop on Transport Network Analysis, no. 8, pp. 165-178, Matsuyama, Japan, 2000.

[22] M. G. H. Bell and J.-D. Schmoker, "Public transport network reliability topological effects," in Proceedings of the 3rd International Conference on Transportation and Traffic Studies, Guanxi People's Press, Guilin, China, 2002.

[23] S. Y. Zhu, W. Wang, W. Deng, Y. Tang, and B. Wang, "Research on traffic network reliability and access road algorithm," China Journal of Highway and Transport, vol. 13, no. 1, pp. 91-94 (Chinese).

[24] J. Jin, "Reliability discussion on urban road network," Journal of Transportation Systems Engineering and Information Technology, vol. 2, p. 76, 2004 (Chinese).

[25] H. Lin, C. Y. Wang, and L. Li, "Study on network travel time reliability under contingent events," Journal of Engineering College of Armed Police Force, vol. 21, p. 63, 2005 (Chinese).

[26] H. Y. Fan, Z. Z. Wu, and X. G. Yang, "Research on the reliability of the topological structure for road transportation networks," Journal of China University of Mining and Technology, vol. 34, no. 4, pp. 482485, 2005 (Chinese).

[27] P. C. Yuan, Y. Han, and W. D. Ma, "Stochastic transportation network equilibrium model based on travel-time reliability," Journal of University of Shanghai for Science and Technology, vol. 30, no. 4, pp. 352-356, 2008 (Chinese). 


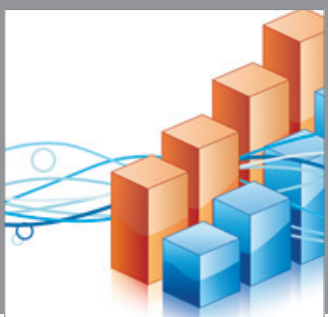

Advances in

Operations Research

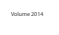

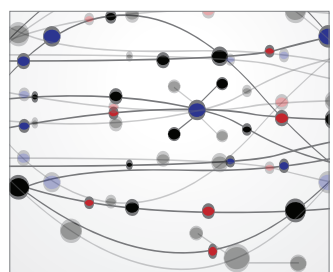

\section{The Scientific} World Journal
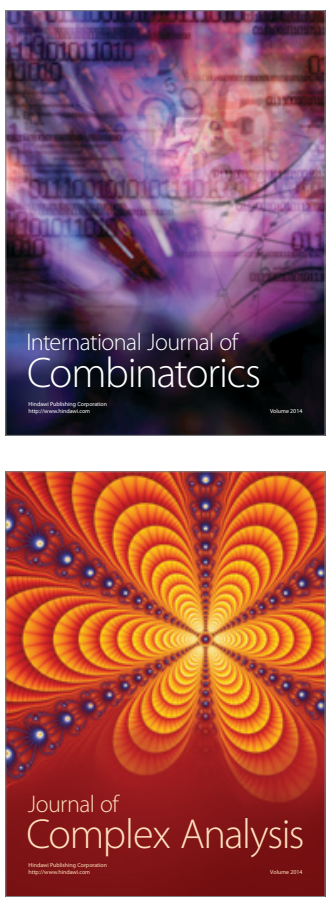

International Journal of

Mathematics and

Mathematical

Sciences
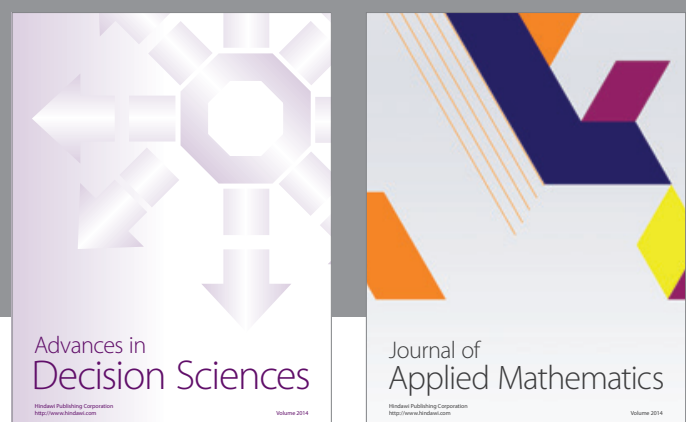

Journal of

Applied Mathematics
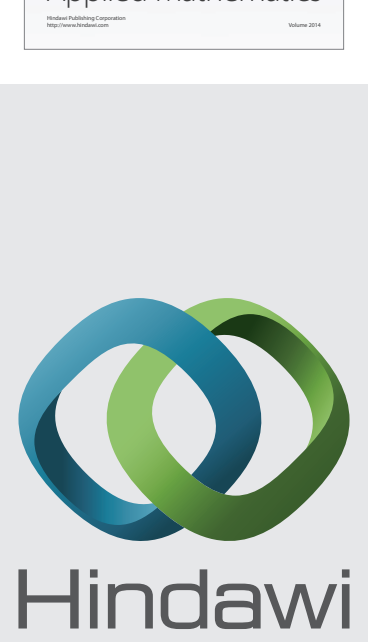

Submit your manuscripts at http://www.hindawi.com
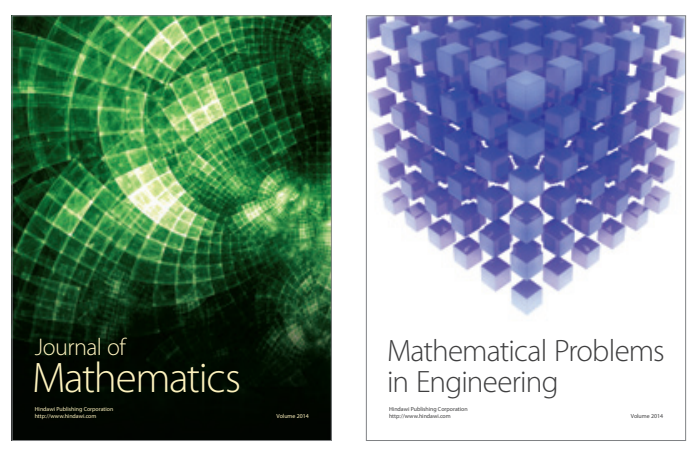

Mathematical Problems in Engineering
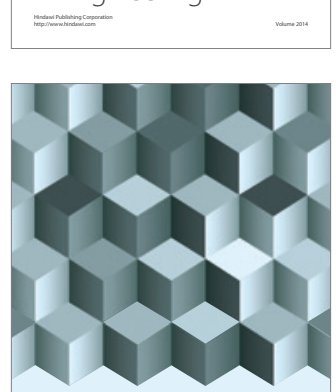

Journal of

Function Spaces
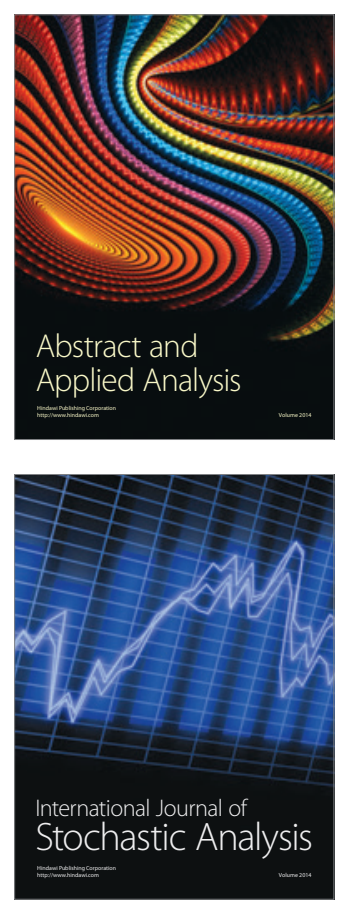

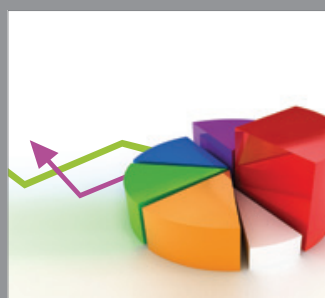

ournal of

Probability and Statistics

Promensencen
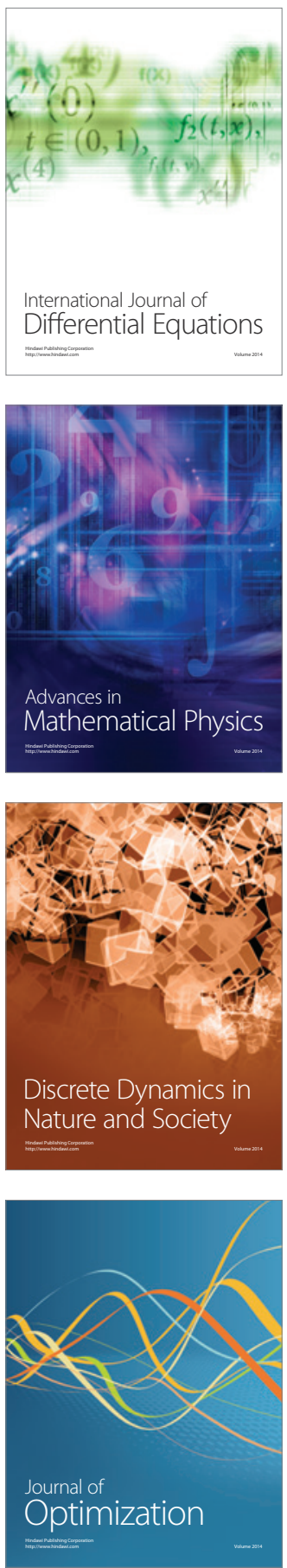